\title{
EFEITOS NEUROMODULADORES DO BROMAZEPAM QUANDO INDIVÍDUOS SÃO EXPOSTOS A UMA TAREFA DE APRENDIZAGEM MOTORA
}

\section{Eletrencefalografia quantitativa (EEGq)}

\author{
José Inácio Salles', Victor Hugo Bastos², Marlo Cunha", \\ Dionis Machado4, Maurício Cagy5, Vernon Furtado6, \\ Luis Fernando Basile? , Roberto Piedade ${ }^{8}$, Pedro Ribeiro ${ }^{9}$
}

\begin{abstract}
RESUMO - Os efeitos sedativos do bromazepam no desempenho cognitivo têm sido amplamente investigados. Várias abordagens têm sido implementadas no intuito de avaliar a influência do bromazepam em sujeitos submetidos à tarefa motora. Neste contexto, o presente estudo objetiva avaliar as alterações eletrofisiológicas em sujeitos expostos à tarefa de datilografia e tratados com bromazepam (6 mg). Os dados do EEGq foram gravados simultaneamente à tarefa. Em particular, a potência relativa na banda delta $(0,5-3,5 \mathrm{~Hz})$ foi analizada. $\mathrm{O}$ tempo de execução e erros durante a tarefa foram considerados variáveis comportamentais. 0 grupo experimental (bromazepam $6 \mathrm{mg}$ ) demonstrou melhor desempenho e valores de potência relativa mais elevados que o grupo controle (placebo). Estes resultados sugerem que menor nível de ansiedade favorece o desempenho motor.
\end{abstract}

PALAVRAS-CHAVE: bromazepam, aprendizagem motora, EEGq, banda delta.

\begin{abstract}
Neuromodulatory effects of bromazepam when individuals were exposed to a motor learning task: quantitative electroencephalography (qEEG)

ABSTRACT - The sedative effects of bromazepam on cognitive and perf o rmance have been widely investigated. A number of different approaches have assessed the influence of bromazepam when individuals are engaged to a motor task. In this context, the present study aimed to investigate electrophysiological changes when individuals were exposed to a typewriting task after taking $6 \mathrm{mg}$ of bromazepam. qEEG data were simultaneously re co rded during the task. In particular, relative power in delta band $(0.5-3.5 \mathrm{~Hz})$ was analyzed. Time of execution and errors during the task were registered as behavioral variables. The experimental group, bromazepam $6 \mathrm{mg}$, showed a better motor perf o rmance and higher relative power than control individuals (placebo). These results suggest that the use of bromazepam reduces anxiety levels as expected and thus, produces an increment in motor performance.
\end{abstract}

KEY WORDS: bromazepam, motor learning, qEEG, delta band.

Experimentos sobre processos de aquisição de habilidades motoras contribuem para o entendimento de como o SNC integra e incorpora diferentes fontes de informação e possíveis variações em enfermidades ou na utilização de medicamentos. O bromazepam atua como um ansiolítico e, em dose mais elevada, pode apresentar efeito hipnótico e miorrelaxante ${ }^{1-4}$. Ele atua como neuromodulador, facilitando a interação do transmissor GABA com seu receptor ${ }^{1}$. Este efeito ocorre em vários níveis do sistema nervoso

\footnotetext{
1'Doutorando, Laboratório de Mapeamento Cerebral e Integração Sensório-Motora, Instituto de Psiquiatria (IPUB), Universidade Federal do Rio de Janeiro, Rio de Janeiro RJ, Brasil (UFRJ); ${ }^{2}$ Doutorando (IPUB/UFRJ), Professor Pesquisador UNIBENNETT e UNIABEU; ${ }^{3}$ Mestrando (IPUB/UFRJ); ${ }^{4}$ Mestranda (IPUB/UFRJ); ${ }^{5}$ D.Sc. Engenharia Biomédica, COPPE - IPUB (UFRJ); ${ }^{6}$ Professor Titular, PROCIHM; ${ }^{7}$ Laboratório de Neurociências (LIM-27), Faculdade de Medicina da Universidade de São Paulo e Faculdade de Psicologia e Fonoaudiologia, Universidade Metodista de São Paulo (UNIMESP), São Paulo SP, Brasil; ${ }^{8}$ Professor Adjunto III Doutor, (IPUB/UFRJ); ${ }^{9}$ Professor Adjunto II PhD, Escola de Educação Física e Desporto (EEFD - Departamento de Biociências da Atividade Física, Laboratório de Mapeamento Cerebral e Integração Sensório-Motora, IPUB, UFRJ, Professor Pesquisador, Universidade Castelo Branco (PROCIMHUCB), Rio de Janeiro RJ, Brasil.
}

Recebido 9 Maio 2005, recebido na forma final 22 Agosto 2005. Aceito 19 Outubro 2005. 
central (SNC) influenciando mecanismos fisiológicos importantes para o processo de aprendizagem motora. Atualmente, uma das formas utilizadas para investigar alterações na atividade elétrica do cére $b$ ro em função de medicamentos é através da técnica de eletrencefalografia quantitativa (EEGq).

Neste estudo, sinais eletrencefalográficos foram registrados durante a execução da tarefa de datilografia, que teve início 40 minutos após o sujeito ingerir bromazepam ou placebo. A atividade de EEGq foi di recionada à banda delta $(0,5-3,5 \mathrm{~Hz})$, um ritmo que de acordo com experimentos prévios expressa baixa atividade no córtex cerebral, associada a estágios de sono e redução dos níveis de ativação durante estado de vigília ${ }^{5}$. Especificamente, a banda delta está presente durante a fase do sono não REM (rapid eye mo viment $)^{6-7}$. O presente estudo verificou a influência do bromazepam durante o aprendizado de uma tarefa de datilografia relacionada à banda delta. Desta fo rma, foi questionado se a diminuição da atividade eletrocortical, devido ao uso de bromazepam, alteraria a perfo rmance motora e se tal alteração estaria associada à atividade na banda delta.

\section{MÉTODO}

Amostra - Foi constituída de 30 estudantes de Graduação e Pós-Graduação dos cursos na área de saúde. Foram considerados critérios de inclusão: ausência de comprometimento da saúde físi ca e mental (anamnese prévia); não usuários de substâncias psicotrópicas ou psicoativas; sono de 6 a 8 horas na noite anterior ao experimento; inexperiência prévia em datilografia, dom inância manual destra de acordo com o inventário de Edinburgh ${ }^{8}$. Quatro sujeitos foram descartados, pois embora tenham relatado inexperiência na tarefa de datilografia apresentaram as variáveis comportamentais (tempo e erro) compatíveis com a de sujeitos experientes (menos de $5 \%$ de erro e/ou tempo inferior a 5 minutos no primeiro bloco da tarefa) ${ }^{9}$. Os erros foram considerados conforme critérios pré-estabelecidos e conferidos por três dos autores.

Os sujeitos foram informados a respeito do objetivo do estudo e assinaram declaração de consentimento na qual a condição experimental encontrava-se detalhadamente descrita. O experimento foi aprovado pela Comissão de Ética do Instituto de Psiquiatria da Universidade Federal do Rio de Janeiro.

Desenho experimental - Ao chegar no laboratório no dia e hora previamente agendados, o sujeito era informado quanto ao procedimento experimental. Após concordar e assinar a declaração de consentimento tinha início a primeira captação de sinal por meio da EEGq. Esta primeira captação consistia em seis minutos nos quais o sujeito mantinhase de olhos fechados e seis minutos de olhos abertos, sendo solicitado a evitar piscada a fim de minimizar a quantidade de artefatos musculares no EEG. Após esta primeira etapa de captação, o sujeito ingeria uma cápsula que poderia conter placebo (amido $400 \mathrm{mg}$ ) ou $6 \mathrm{mg}$ de bromazepam. A distribuição das cápsulas seguiu um modelo duplo-cego randomizado para evitar que houvesse influência nos resultados por parte dos examinadores. Trinta minutos após a ingestão da cápsula era feita uma avaliação do nível de atenção do sujeito pelo teste de Stroop. Após o teste era iniciada nova captação, desta vez simultaneamente à tarefa da datilografia. Ao término da tarefa era realizada mais uma etapa de captação (seis minutos olhos fechados e seis minutos olhos abertos).

Aquisição de dados - Para a captação do sinal eletrence falográfico foi utilizado o aparelho Braintech 3000 (EMSA - Instrumentos Médicos, Brazil), sistema que utiliza uma placa conversora analógica-digital (A/D) de 32 canais com resolução de 12 bits, colocada em um slote ISA de um Pentium III - com um processador de $750 \mathrm{~Hz}$. Foi utilizada uma touca que continha os eletrodos dispostos de acordo com o sistema internacional $10-20^{10}$, incluindo-se os eletro $\mathrm{d}$ o $\mathrm{s}$ de referência posicionados nos lóbulos das orelhas (bi-auricular) ${ }^{11}$. A sala utilizada para captação do sinal eletrencefalográfico foi preparada com isolamento acústico e elétrico, durante a aquisição do sinal as luzes foram reduzidas, exceção feita ao momento da tarefa.

O sinal adquirido em determinado eletrodo foi resultante da diferença entre o potencial elétrico dele no escalpo e a referência pré-estabelecida. De início, foram verificados os níveis de impedância de cada eletrodo, cujos valores foram fixados entre $5-10 \mathrm{~K}$ ohms $(\Omega)$ e mantidos nesses padrões. Os sinais adquiridos tinham um total de amplitude (pico a pico) menor que $100 \mu \mathrm{V}$. Por este motivo, o sinal foi amplificado com ganhos de 20000 vezes. Os sinais eletrencefalográficos adquiridos flutuaram entre 0,01 e $50 \mathrm{~Hz}$. A atividade elétrica ocular foi estimada com a colocação de dois eletrodos de $9 \mathrm{~mm}$ de diâmetro montados de forma bipolar. Os eletrodos foram posicionados, respectivamente, acima e abaixo da órbita do olho direito para registrar movimentos oculares verticais e no canto externo (canthus) dele.

Os parâmetros do EEGq foram extraídos de trechos de 2 segundos antes e depois do início do movimento em 20 derivações de acordo com o sistema 10-20 de posicionamento de eletrodos. Trechos de sinal contaminados por a rtefatos musculares foram retirados por um algoritmo de rejeição automática de artefatos por trespasse de limiar $(100 \mu \mathrm{V})$. A partir da estimativa da densidade espectral de potência, obteve-se a potência nas bandas do EEG (delta, teta, alfa e beta) antes do início do movimento (entre $-2 \mathrm{~s}$ e 0 s, considerado como referência - R) e após o movimento (entre 0 s e $2 \mathrm{~s}$, denominado $\mathrm{P}$ ), com relação a cada pressiona mento de tecla de interesse. Em 0 s tem-se a representação do momento exato do pressionamento da tecla de interesse. Além da potência em cada banda, a potência total também foi estimada visando à obtenção da potência relativa, definida como a razão entre a potência em cada banda e a potência total. Com o objetivo de facilitar a visualização, todos trechos de sinal contidos entre $2 \mathrm{~s}$ antes e $2 \mathrm{~s}$ após o p ressionamento da tecla de interesse sofreram filtragem passa-faixa (associação de filtros passa-altas e passa-baixas 
Butterworth de ordem 2 cada). Uma vez filtrados, os respectivos trechos de dados foram elevados ao quadrado e sofreram filtragem passa-baixas objetivando a detecção de envoltória. Uma promediação de todas as trilhas ocorre u para se obter à evolução temporal da potência média em todas as $\mathbf{2 0}$ derivações utilizadas, que pode ser interpretado visualmente e servir de suporte às conclusões estatísticas.

Procedimento da tarefa motora - Para a realização da tarefa optou-se por máquina de datilografia de modelo antigo (Olivetti modelo Línea 98); tal opção se deve ao fato da re $p$ resentação cortical ser maior quanto maior o grau de força empregada na tarefa ${ }^{12-14}$. Os sujeitos estavam confortavelmente sentados em cadeira com suporte para os antebraços a fim de minimizar os artefatos musculares. A distância entre a cadeira do sujeito e a mesa onde se encontrava a máquina de datilografia foi de cerca de $20 \mathrm{~cm}$ variando em função do comprimento do antebraço de cada sujeito. O teclado da máquina foi coberto com uma caixa de madeira que evitava a visualização do posicionamento das mãos sobre o teclado e que exigia do sujeito a criação de uma "referência espacial" para o teclado.

A tarefa consistiu num método de datilografia ${ }^{9} \mathrm{com}$ aprendizagem progressiva e realizada num único dia. $\mathrm{O}$ exercício proposto era composto por quatro blocos sendo cada bloco constituí do de 12 linhas. Cada linha continha cinco seqüências de letras para cada mão. A seqüência de letras para cada mão era assim estabelecida: asdfg para mão esquerda e çlkjh para mão direita. A seqüência da mão esquerda era iniciada pelo dedo mínimo pressionando a tecla re f e rente à letra $a$, em seguida ocorria o pressi on amento da tecla $s$ pelo anular, tecla $d$ pelo dedo médio, tecla $f$ e $g$ pelo indicador. Terminada a seqüência da mão esquerda era efetuado o pressionamento da tecla espaço, utilizando para isto o polegar esquerdo ou o direito, e então era iniciada a seqüência da mão direita (dedo mínimo pressionando ç, anular na tecla $l$, médio para $k$, indicador para as teclas $j$ e $h$ ).

Abaixo do teclado da máquina de datilografia existia um circuito elétrico responsável pela captação do pressionamento das teclas de interesse, a saber, a e $g$, pois respectivamente representavam início e término da seqüência da mão esquerda, ç e $h$ pelo mesmo motivo em função da mão direita e a tecla espaço que representava mudança para seqüência seguinte. Tal circuito era composto por cinco dispositivos em forma de "U" denominados optoeletrônicos. Cada optoeletrônico possui uma porção emissora e uma porção receptora de luz que permitia a transferência da informação de pressionamento das teclas de interesse para o sistema de aquisição de dados onde eram efetivamente reconhecidas as letras conforme seus traçados gráficos característicos (mediante passagem por um simples conversor digital-analógico).

Bandas de freqüência e localização espacial - Os dados eletrofisiológicos de interesse referem-se à banda delta. A escolha da banda delta justifica-se pelo fato desta estar relacionada a mecanismos de sono/vigília ${ }^{15}$. Estudos com ansiolíticos e ervas usadas para distúrbios do sono, com o
Kava Kava, apontam para uma tendência de aumento da banda delta em diversas áreas corticais ${ }^{11,16}$.

Áreas frontais foram selecionadas em virtude da relação destas aos mecanismos de motivação, planejamento e execução de movimentos voluntários. As áreas centrais, por representarem o córtex sensório-motor primário, que tanto recebe informações somáticas, como controlam movimentos dos membros. A inserção dos eletrodos parietais justificase pelo controle dos mecanismos sensoriais e espaciais ocorre rem em tais áre a s ${ }^{17-18}$. Os eletrodos foram enquadrados dentro de quatro setores: anterior esquerdo $A E$ (eletrodos F3, C3 e T3), anterior direito AD (eletrodos F4, C4 e T4), posterior esquerdo PE (eletrodos P3, T5 e 01) e posterior direito PD (eletrodos P4, T6 e O2).

Análise estatística - Em relação ao teste de Stroop foi realizado um teste-t (grupos independentes) para tempo e escore bruto. Em especial, o Stroop foi realizado 30 minutos após a ingestão do placebo ou bromazepam $6 \mathrm{mg}$, dependendo da randomização. Particulamente, este teste tem sido utilizado para análise dos processos de atenção em modelos com eletrencefalografia ${ }^{18}$.

Para análise comportamental, tempo de execução e erros cometidos na tarefa, foi utilizado o teste ANOVA (Two-Way) com relação a cada uma das duas variáveis. Em especial, ANOVA foi implementada para comparar dois fatores: condição (placebo e bromazepam $6 \mathrm{mg}$ ) e bloco (blocos I, II, III e IV).

A análise dos dados eletrofisiológicos considerou a ta refa completa e não a subdivisão em blocos considerada na análise comportamental. Os valores de potência relativa obtidos (dados eletrofisiológicos) 2 segundos antes do p ressionamento da tecla $h$, e 2 segundos após o pressiona mento, foram transformados para logaritmo em base natural $(\log N)$ utilizando osoftware SPSS versão 10.0. Esta transformação se fez necessária porque os valores de potência relativa não apresentam distribuição tipo Gauss. A análise dos dados por histograma e pelo teste de Kolmogorov Smirnov mostraram normalidade dos dados após passagem para logaritmo natural ${ }^{19}$. Em relação aos dados eletrofisiológicos, o teste ANOVA (Three-Way) comparou os fatores: condição (controle $x$ experimental); momento (dois segundos antes do pressionamento da tecla $h$, e dois segundos após o pressionamento da tecla); e setores no escalpo ( $A E, A D, P E$, e PD). Para todas as análises foi considerado um $p$ estatístico menor ou igual a 0,05 $(p \leq 0,05)$.

\section{RESULTADOS}

Variável neuropsicológica (atenção) - O teste utilizado para verificar nível de atenção (teste de Stroop) não mostrou diferença significativa entre as condições placebo e bromazepam, tanto para a variável tempo de execução do teste $(p=0,976)$, quanto para escore bruto atingido pelos participantes $(p=0,332)$.

Variáveis comportamentais - Os resultados obtidos para a variável número de erros apresentaram efeitos principais para os fatores condição $(p=0,021)$ e blocos 


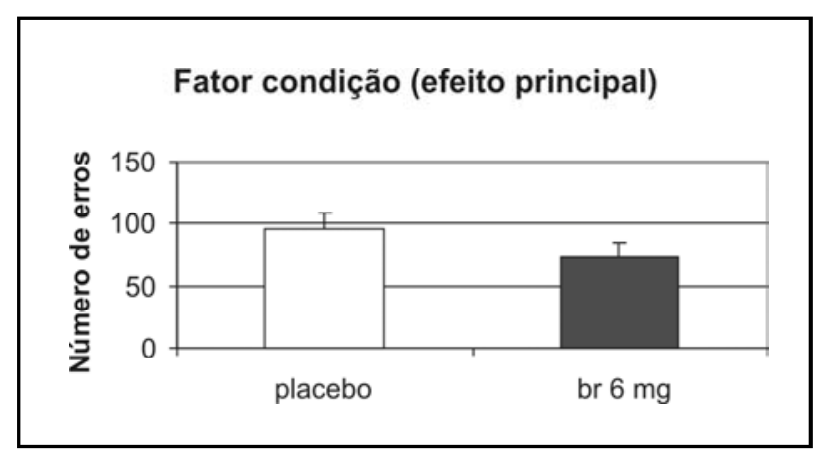

Fig 1. Média do número de erros durante a tarefa de datilogra fia, comparando as condições placebo e bromazepam (6 mg).

$(p=0,011)$. Quanto ao primeiro fator, o número de erros durante a tarefa inteira cometido por cada condição (Fig 1), os resultados são: placebo (média=96,5; $\mathrm{S} \pm 55,1$ ) e bromazepam (média $=73,2 ; \mathrm{S}_{ \pm} 48,6$ ). Em relação ao segundo fator, blocos (Fig 2), os resultados são: bloco 1 (média=112,4; $\mathrm{S} \pm 67,8$ ), bloco 2 (média= 85,$1 ; \mathrm{S} \pm 45,1$ ), bloco 3 (média $=69,7 ; \mathrm{S} \pm 46,3$ ) e bloco 4 (média $=72,1 ; S_{ \pm} 40,1$ ). As diferenças entre blocos atingiram significância estatística somente entre o bloco $1 \mathrm{x}$ bloco 3 e bloco 1 x bloco 4 .

A análise da variável tempo de execução, medida em segundos (Fig 3) relata um efeito principal somente para o fator bloco $(p=0,000)$. Aqui foram obtidos os seguintes dados: bloco 1 (média $=543,1 ; \mathrm{S} \pm 176,6$ ), bloco 2 (média=434,9; $\mathrm{S} \pm 105,0$ ), bloco 3 (média=392,9; $\mathrm{S} \pm 88,1$ ) e bloco 4 (média=373,3; $\mathrm{S}_{ \pm} 78,3$ ). Para a referida variável foram encontradas diferenças significativas estatisticamente sempre que se comparou o bloco 1 com qualquer dos outros blocos. Todavia, não houve significância entre o bloco 2 x bloco 3 , bloco $2 \times$ bloco 4 ou bloco 3 x bloco 4 .

Variável eletrofisiológica - Foi verificada uma interação entre os três fatores: condição, momento e se-

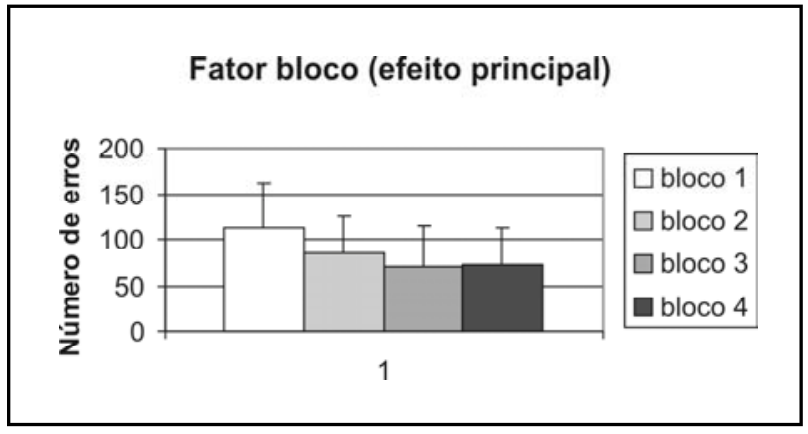

Fig 2. Média do número de erros dentro de cada bloco da tarefa de datilografia.

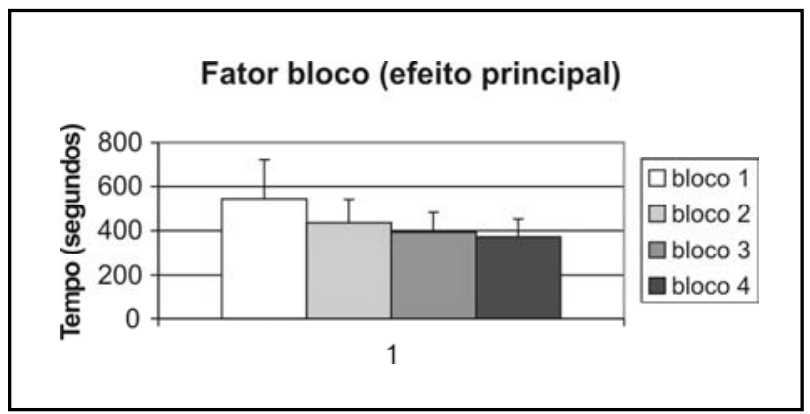

Fig 3. Tempo transcorrido durante a execução de cada bloco da tarefa de datilografia.

tor (Fig 4) que apresentou significância estatística $(p=0,002)$. Comparando os valores de potência relativa refe rentes a cada um dos quatro setores do escalpo, no momento pré-movimento, foram obtidos os seguintes resultados: placebo ( $A E$ : média $=-3,288$; $S \pm 0,214)$, ( $A D:$ média $=-3,287 ; S \pm 0,185)$, (PE: média= $\left.-3,096 ; S_{ \pm} 0,129\right)$ e (PD: média $=-3,073 ; S \pm 0,017$ ); bromazepam ( $A E$ : média $=-2,806 ; S \pm 0,011)$, ( $A D$ : média $=-2,760 ; \mathrm{S} \pm 0,013$ ), ( $P E:$ média $=-2,716 ; \mathrm{S} \pm 0,012$ ) e (PD: média $=-2,674 ; S \pm 0,012$ ). Para o referido intenalo de tempo (pré-movimento) a condição pla-

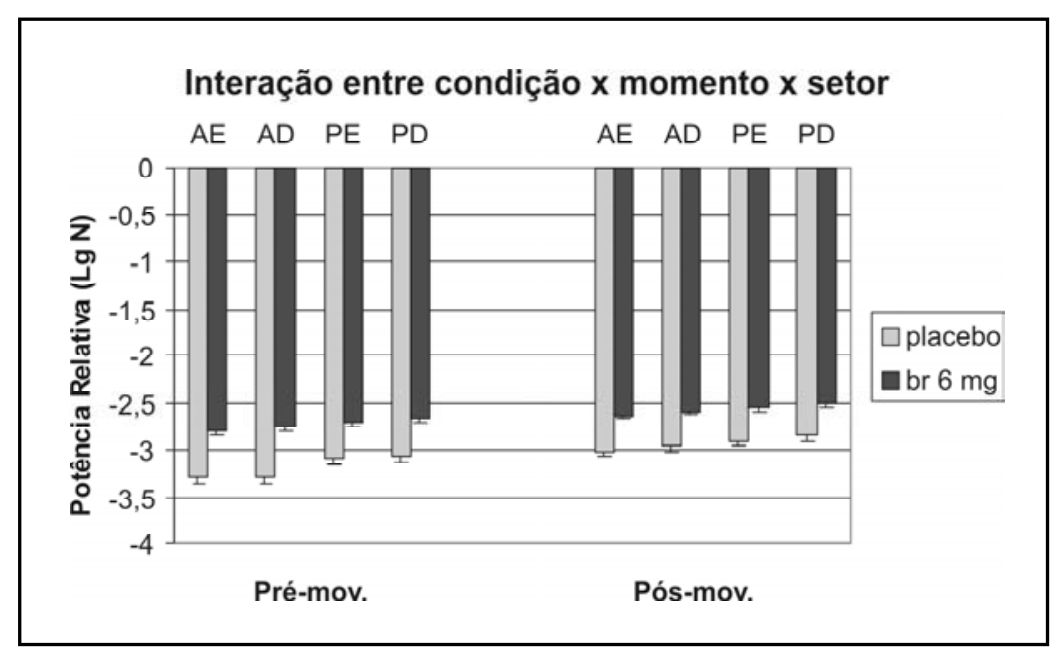

Fig 4. Potência Relativa para a banda delta. Os dados são apresentados para as condições pla cebo e bromazepam $6 \mathrm{mg}$ (br $6 \mathrm{mg}$ ) referentes aos setores do escalpo anterior esquerdo (AE), anterior direito $(A D)$, posterior esquerdo (PE) e posterior direito $(P D)$. Os valores de potência foram obtidos comparando os momentos pré e pós-movimento (pressionamento da tecla $h$ com o dedo indicador direito). 
cebo apresentou valores médios de potência mais negativos do que a condição bromazepam. Ambas as condições apresentaram maior negatividade de valorespara os setores anteriores (AE e AD) em comparação com os posteriores ( $P E$ e PD), porém, essa diferença foi mais relevante na condição placebo.

A mesma comparação entre os valores de potência, dentro de cada setor, entre as condições, quando o b s e rada no momento pós-movimento apresentou os seguintes resultados: placebo ( $\mathrm{AE}$ : média $=-3,014$; $S \pm 0,017)$, ( $A D:$ média $=-2,958 ; S \pm 0,016)$, (PE: média= $-2,905 ; \mathrm{S} \pm 0,015)$ e (PD: média $=-2,854 ; \mathrm{S} \pm 0,014$ ); bromazepam (AE: média $=-2,634 ; S \pm 0,011$ ), (AD: média $=-2,595 ; S \pm 0,011)$, (PE: média $=-2,558 ; S \pm 0,010$ ) e (PD: média $\left.=-2,522 ; S_{ \pm} 0,010\right)$. Também nesse período de tempo (após o início do movimento) a condição placebo apresentou valores de potência mais negativos do que a condição bromazepam. Para ambas as condições os setores anteriores do escalpo continuaram expressando maior negatividade de seus valores quando comparados com os setores posteriores. Todavia, essas diferenças seguiram um mesmo padrão para as duas condições, não apresentando tal relevância como a observada no grupo bromazepam no período pré-movimento.

\section{DISCUSSÃO}

Variável neuropsicológica (atenção) - Os resulta dos a partir do teste de Stroop demonstraram não haver prejuízos nos processos de atenção, devido à influência do medicamento, minutos antes de iniciarem a tarefa motora. A droga, na dose utilizada, parece não influenciar nos mecanismos de atenção. Existe a possibilidade de tal teste não ser sensível para captar mudanças atencionais ocorridas em função do uso de medicamentos. Em modelos futuros pretendese a associação do teste de Stroop a outros testes neuropsicológicos a fim de observar parâmetros que possam modificar os níveis primários de atenção, assim como outras funções superiores que parecem estar envolvidas.

Variáveis comportamentais (tempo e erros) - A análise dos dados comportamentais, a partir das variáveis erro e tempo, evidenciou que tanto os sujeitos que receberam placebo quanto os tratados com bromazepam obtiveram graus significativos de melhora quando se comparou o primeiro e o último bloco da ta refa. A transição do primeiro bloco para o segundo pareceu coincidir com algum estágio crítico da aprendizagem observada, talvez marcando a evolução de um estágio com processamento controlado (com ênfase em demandas cognitivas), em direção a estágios mais avançados aproximando-se do automatismo (com predomínio de demandas de natureza motora p ropriamente dita). A ausência de significância estatística entre os blocos subseqüentes ao primeiro sugere que seja aumentado o volume de trabalho dentro de cada bloco, a fim de fornecer um intervalo de tempo suficiente para que a evolução atinja significância estatística. As diferenças entre as condições placebo e bromazepam observadas para número de erros, mas não para tempo de execução, sugerem melhor desempenho da condição bromazepam ${ }^{1,20-22}$. Estes se tornaram mais precisos com relação ao pressionamento das teclas datilográficas, porém, não mais velozes quando comparados à condição placebo. Tais achados podem estar associados ao efeito do medicamento que ao "reduzir" os níveis de ativação e ansiedade ocasionados pela situação de tarefa, acabou por favorecer a incorporação e aprimoramento dos mecanismos próprios do controle motor. Em outras análises, sujeitos que foram submetidos a testes de atenção, extensão de memória e tempo de reação mediante uso de diferentes doses de bromazepam não demonstraram prejuízo psicomotor tanto em função da droga quanto em função da dosagem ${ }^{21}$.

Variáveis eletrofisiológicas - Quanto à análise eletrencefalográfica, a interação entre os fatores condição, momento e setor demonstraram que sujeitos sob a condição placebo apresentaram valores de potência relativa na banda delta mais negativos do que os valores apresentados pela condição bromazepam. Isso se fez presente tanto para o momento pré quanto para o pós-movimento. Contudo, no momento pré (preparação motora), a condição placebo demonstrou diferença mais relevante ao comparar setores anteriores versus setores posteriores no escalpo. Esse padrão de diferença não foi verificado para a condição bromazepam no momento pré-movimento, nem para nenhuma das duas condições quando em momento pós-movimento (execução motora). Tais achados podem estar associados ao maior nível de ativação em sujeitos sob a condição placebo, em especial no período pré-movimento quando os setores mais ativos ( $A E$ e $A D$ ) são os representativos de áreas envolvidas com as emoções, planejamento, motivação e preparação motora. Trabalhos têm mostrado que a banda de freqüência delta esta relacionada aos estágios de sono não $\mathrm{REM}^{6,7}$. O u t ros estudos mostraram que a banda delta tende a aumentar com sedativos, ansiolíticos e hipnóticos e a diminuir com psicoestimulantes ${ }^{23,24}$. Tais relatos estão de acordo com os presentes resultados os quais demonstraram valores médios de potência em delta maiores na condição bromazepam. 
A observação de maior atividade no setor $A E$ seguido pelo setor $A D$ reflete importante engajamento das áreas de planejamento para a realização da tarefa. Adicionalmente, o setor AE abrangeu também as áreas re presentativas do controle motor da mão e dedos dire i t o s $^{25-27}$, os quais foram assumidos neste estudo como movimentos de interesse. A maior incidência de delta no setor $\mathrm{AE}$ ocorreu para a condição bromazepam, e isso pode estar associado a um estado de maior "relaxamento" mental, induzido pelo medicamento, que até uma certa dosagem, tende a favorecer a performance do indivíduo. Em contrapartida, a condição placebo apresentou valores mais negativos de potência, que sugerem ter despendido maior esforço mental, em especial durante a fase de planejamento motor.

Em conclusão, sob doses relativamente baixas o b romazepam não parece causar prejuízo para a performance de indivíduos em situação de aprendiza gem motora. Contrariamente, o uso do medicamento p a rece ter favorecido o rendimento por evitar níveis indesejáveis de ativação e ansiedade inerente à aprendizagem.

\section{REFERÊNCIAS}

1. GraeffF. Drogas psicotrópicas e seu modo de ação. 2.Ed. São Paulo: EPU, 1989.

2. Leeuwen Van TH, Verbaten MN, Koelega HS, Kenemans JL, Slangen JL. Effects of bromazepam on single-trial event-related potentials in a visual vigilante task. Psychopharmacology 1992;106:555-564.

3. Versiani M, Nardi AE, Figueira I, Mendlowicz M, Marques C. Doubleblind placebo controlled trial with bromazepam in social phobia. J Brasil Psiquiatria 1997;46:167-171.

4. Blanco C, Antia SX, Liebowitz MR. Pharmacotherapy of social anxiety disorder. Biol Psychiatry 2002;51:109-120.

5. Puente-Muñoz AI,Martínez DAP, Valderrey IV. El papel del sueño de ondas lentas en la regulación homeostática del sueño. Rev Neurol 2002; 34:211-215.

6. Marquez JM, Carrier J, Decary A, et al. Slow-wave sleep and delta power in rapid eye movement sleep behavior disorder. Ann Neuro 1 2004;57:277-282.

7. Maltez J, Hyllienmark L, Nikulin VV, Brismar T. Time course and variability of power in different frequency bands of EEG during resting conditions. Neurophysiologie Clinique 2004;34:195-202.
8. Oldfield R. The assessment and analysis of handedness: the Edinburgh inventory. Neuropsycology 1971;9:97-113.

9. Santos C. Novíssimo guia do datilógrafo. 40.Ed. São Paulo: Saraiva, 1997:5-7.

10. Jasper $H$. The ten-twenty electrode system of the international federation. EEG Clin Neurophysiol 1958;10:371-375.

11. Niedermeyer E, Silva F. Electroencephalography: basic principles, clinical applications and related fields. 4.Ed. Baltimore-Munich: Urban \& Schwarzenberg, 1999.

12. Hamzei F, Dettmers C, Rzanny R, Liepert J, Büchel C, Weiller C. Reduction of excitability ("inhibition") in the ipsilateral primary motor cortex is mirro red by fMRI signal decreases. Neuroimage $2002 ; 17$ : 490-496.

13. Slobounov S, Chiang H, Johnston J, Ray W. Modulated cortical control of individual fingers in experienced musicians: an EEG study. Clin Neurophysiol 2002; 113:2013-2024.

14. Parsons LM, Sergent J, Hodges DA, Fox PT. The brain basis of piano performance. Neuropsychologia 2005;43:199-215.

15. Duckrow RB, Zaveri HP. Coherence of the electroencephalogram during the first sleepcycle. Clin Neurophysiol 2005;116:1088-1095.

16. Shinomiya $K$, Inoue $T$, Utsu $Y$, et al. Effects of kava-kava extract on the sleep-wake cycle in sleep-disturbed rats. Psychopharmacology 2005; in press.

17. Smyrnis N, Theleritis C, Evdokimidis I, Müri RM, Karandreas N. Singlepulse transcranial magnetic stimulation of parietal and prefrontal areas in a memory delay arm poiting task. J Neurophysiol 2003;89:3344-3350.

18. Kandel E, Schwartz S, Jessel T. Principles of neuroscience. 4.Ed. New York: McGraw-Hill, 2000.

19. Schack B, Chen CAN, Mescha S, Witte, H. Instantaneous EEG coherence analysis during the Stroop task. Clin Neurophysiol 1999;110:1410-1426.

20. Hegener O, Jordan R, Haberlein H. Benzodiazepine binding studies on living cells: application of small ligands for fluorescence correlation spectroscopy. Biol Chem 2002;383:1801-1807.

21. Oelschlager H. Chemical and pharmacologic aspects of benzodiazepines. Schweiz Rundsch Med Prax 1989;78:766-772.

22. Hobi V, Dubach UC, Skreta M, Forgo I, Riggenbach H. The subacute effect of bromazepam on psychomotor activity and subjective mood. J Intern Med Res 1982;10:140-146.

23. Dong HS, Dong WS, Duk IJ, Eun HH. Effects of methylphenidate on quantitative EEG of boys with attention-deficit hyperactivity disorder in continuous performance test. Yonsei Med J 2005;46:34-41.

24. Bastien CH, LeBlanc M, Carrier J, Morin CM. Sleep EEG power spectra, insomnia, and chronic use of benzodiazepines. Sleep 2003;26:313-317.

25. Bastos VHV, Alves HVD, Piedade RAM, Silva VF, Silva APRS. Alterações corticais produzidas em função de uma tarefa de datilografia. Fitness Perform 2002;1:53-58.

26. Cunha M, Bastos VHV, Veiga H, Cagy M, Piedade R, Ribeiro P. Alterações na distribuição de potência cortical em função da consolidação da memória no aprendizado de datilografia. A rq Neuropsiquiatr 2004; 62:662-668.

27. Muthukumaraswamy SD, Johnson BW. Changes in rolandic mu rhythm during observation of a precision grip. Psychophysiology 2004; 41:152156 